\title{
Periungual Papules in an Elderly Woman
}

Stacy Y. Kasitinon, MD, PhD; Jennifer A. Day, MD; Travis W.Vandergriff, MD; Jennifer G. Gill, MD, PhD

Eligible for 1 MOC SA Credit From the ABD

This Photo Challenge in our print edition is eligible for 1 self-assessment credit for Maintenance of Certification from the American Board of Dermatology (ABD). After completing this activity, diplomates can visit the ABD website (http://www.abderm.org) to self-report the credits under the activity title "Cutis Photo Challenge." You may report the credit after each activity is completed or after accumulating multiple credits.

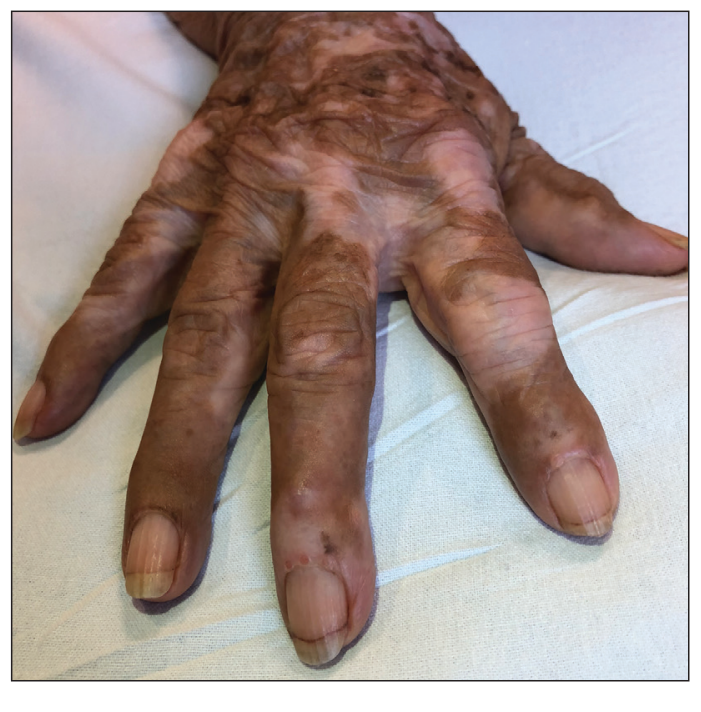

A 79-year-old woman presented with pruritic papules and plaques on the chest, back, arms, hands, legs, and feet of 1 year's duration. She reported a history of hypothyroidism, arthritis, and vitiligo but denied a history of cancer. Physical examination showed pink papules coalescing into plaques on the upper chest and lower back as well as lichenified plaques on the forearms and knees. Erythematous papules on the proximal nail folds of the right first and second digits also were noted. Multiple depigmented patches on the hands, wrists, arms, and lower back also were present, and deformities of the hands and bulbous-appearing knees were observed. Results from a complete blood cell count and blood chemistry analyses showed mild anemia but were otherwise normal. Radiography of the right knee showed degenerative changes and periarticular radiolucencies consistent with an inflammatory arthropathy. A 4-mm punch biopsy specimen from the back was obtained for histopathologic examination.

\section{WHAT'S YOUR DIAGNOSIS?}
a. dermatomyositis
b. erythema elevatum diutinum
c. multicentric reticulohistiocytosis
d. rheumatoid arthritis
e. sarcoidosis

From the Department of Dermatology, University of Texas Southwestern Medical Center, Dallas. Dr. Day also is from the Department of Dermatology, University of Colorado, Aurora. 


\section{THE DIAGNOSIS:}

\section{Multicentric Reticulohistiocytosis}

\section{T} he patient presented with pink papules coalescing into plaques on the upper chest and lower back (Figure 1) as well as a characteristic finding of periungual papules with a coral bead appearance. Histopathologic examination revealed a dense infiltrate of epithelioid histiocytes with amphophilic ground-glass cytoplasm in a nodular configuration (Figure 2). This pattern in conjunction with the clinical features seen in our patient was consistent with a diagnosis of multicentric reticulohistiocytosis (MRH) ${ }^{1-3}$ The cutaneous symptoms were managed with triamcinolone ointment $0.1 \%$ twice daily and oral hydroxyzine $10 \mathrm{mg} 3$ times daily as needed for itching with moderate improvement. She was referred to rheumatology for arthritis management, and the initial cancer screening was negative.

Multicentric reticulohistiocytosis is a rare granulomatous disease characterized by papulonodular cutaneous lesions and severe erosive arthritis. It has an insidious onset and most commonly affects middle-aged women. ${ }^{1}$ Multicentric reticulohistiocytosis typically presents as rounded pruritic papules or nodules that may be pink, red, or brown primarily affecting the face and distal upper extremities. ${ }^{1,3}$ Mucosal involvement occurs in more than half of patients and is characterized by multiple erythematous papules and nodules on the oral and nasopharyngeal mucosae that rarely can produce leonine facies. ${ }^{2} \mathrm{~A}$ hallmark feature of $\mathrm{MRH}$ is the presence of multiple shiny erythematous papules along the proximal and lateral nail folds that take on a coral bead appearance. ${ }^{1,3,4}$ Furthermore, nail changes such as atrophy, longitudinal ridging, brittleness, and hyperpigmentation can occur secondary to a synovial reaction that disturbs the nail matrix. ${ }^{.5}$

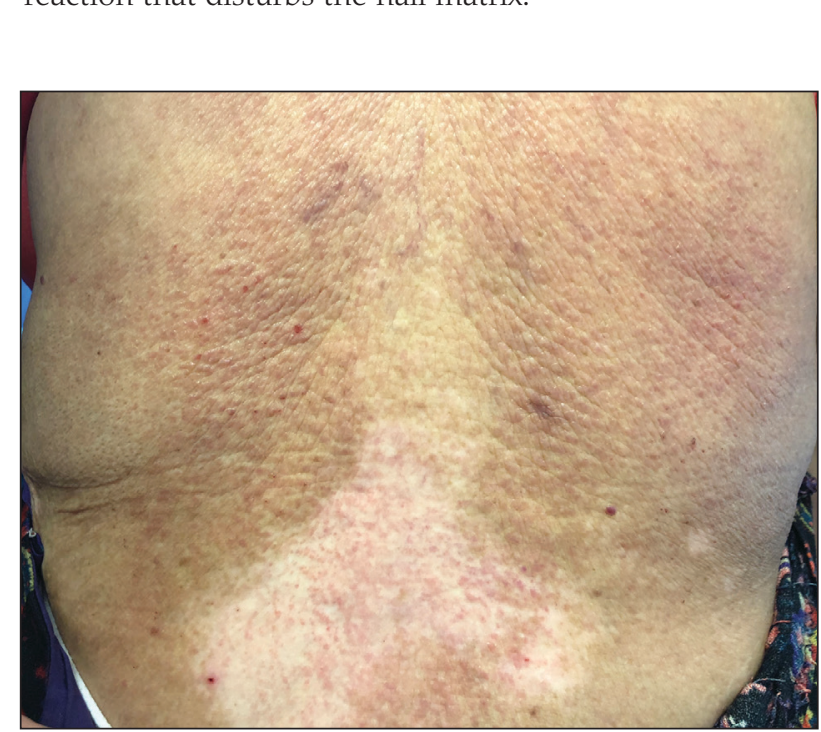

FIGURE 1. Pink papules coalescing into plaques on the lower back.
Joint involvement precedes cutaneous involvement in most cases of MRH. ${ }^{1,5}$ Multicentric reticulohistiocytosis is associated with a symmetric destructive arthritis affecting the hands, knees, shoulders, and hips that often is associated with pain, stiffness, and swelling. ${ }^{1,3}$ The arthritis rapidly progresses in the early stages of the disease but then becomes less active over the subsequent 8 to 10 years. ${ }^{1}$ It has the potential to develop into arthritis mutilans, an end-stage form of arthritis also seen in psoriatic and rheumatoid arthritis that leads to severe joint deformity and debilitation. ${ }^{1,2}$

The etiology of MRH still is unknown, but it has an association with underlying malignancy in up to
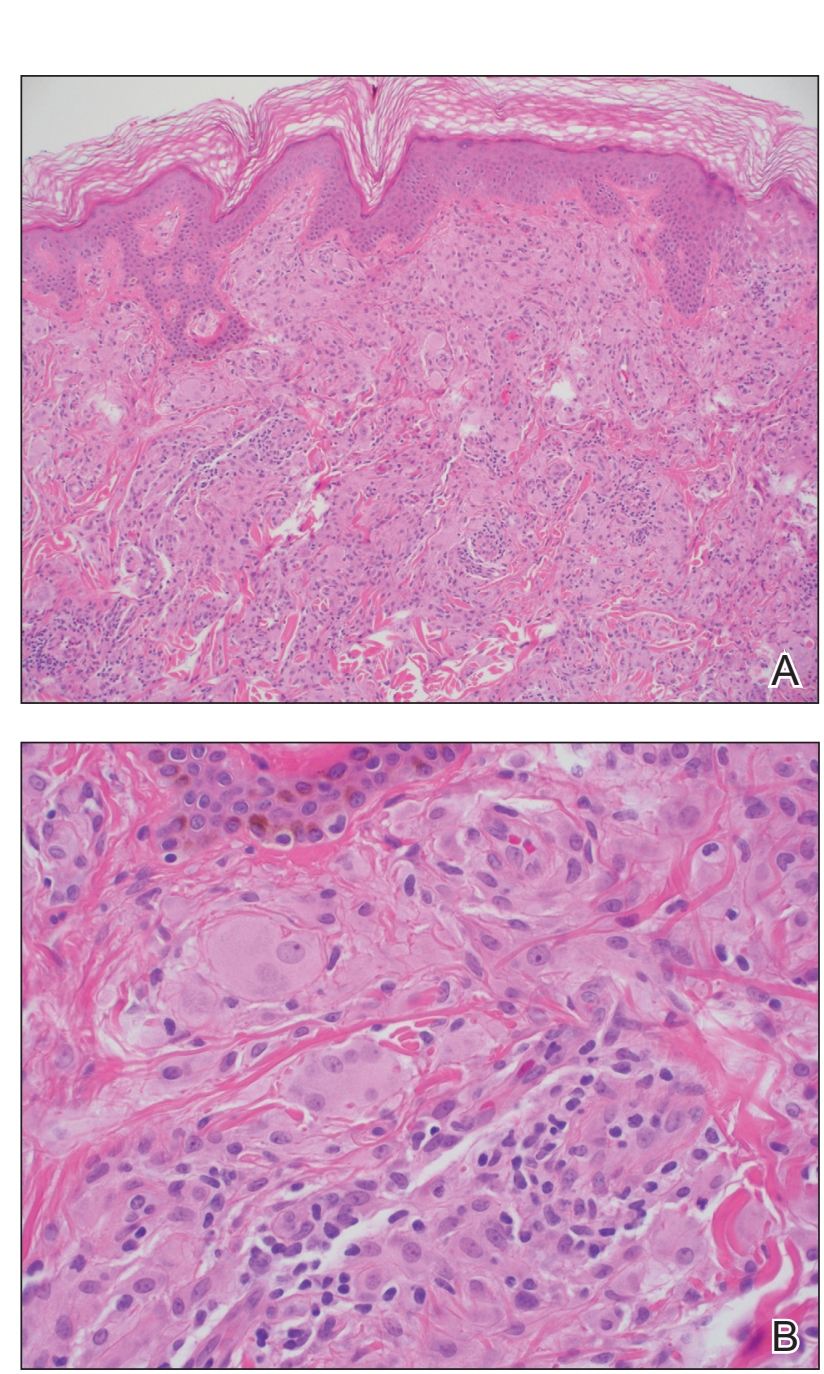

FIGURE 2. A and B, Lesional histopathology showed dermal histiocytic infiltration with multinucleated giant cells containing two-toned, ground-glass cytoplasm and prominent nucleoli (H\&E, original magnifications $\times 40$ and $\times 200$ ) 
$25 \%$ of patients. ${ }^{6}$ Multicentric reticulohistiocytosis has been reported in the context of a wide variety of malignancies including melanoma; sarcoma; lymphoma; leukemia; and carcinomas of the breast, colon, and lung. In some cases, the diagnosis of MRH may even precede the diagnosis of cancer. ${ }^{3}$ Multicentric reticulohistiocytosis also may be associated with autoimmune conditions, ${ }^{3}$ as seen in our patient who had a history of both hypothyroidism and vitiligo.

Histopathologic examination is essential in distinguishing $\mathrm{MRH}$ from other autoimmune disorders associated with hand lesions, rash, and arthralgia. Erythema elevatum diutinum is associated with symmetric, violaceous, red or brown papules and plaques located on the extensor surfaces of the extremities and hands; however, histology reveals a leukocytoclastic vasculitis with a mixture of polymorphonuclear leukocytes and lymphocytes. ${ }^{7}$ Dermatomyositis may present with arthralgia, flattopped, erythematous (Gottron) papules localized over the proximal interphalangeal and distal interphalangeal joints, as well as proximal nail findings. The latter generally presents with periungual erythema associated with dilated capillary loops rather than the discrete orderly papules seen in MRH. Histologic examination of dermatomyositis shows mild epidermal atrophy, vacuolar changes in the basal keratinocyte layer, and a dermal perivascular lymphocytic infiltrate. ${ }^{8}$ Because MRH initially can present with joint symptoms and hand nodules, it may be confused with rheumatoid arthritis. However, rheumatoid arthritis typically is associated with severe osteopenia and tends to affect the metacarpophalangeal and proximal interphalangeal joints rather than the distal interphalangeal joints that most often are affected in MRH. ${ }^{1}$ Histologic examination of rheumatoid nodules reveals palisading granulomas surrounding a central area of fibrinoid necrosis. ${ }^{9}$ Sarcoidosis is a multisystem disease that can present with cutaneous involvement including erythema nodosum, skin plaques, subcutaneous nodules, and papular eruptions in addition to joint lesions. ${ }^{10}$ Sarcoidosis most frequently involves the lungs, manifesting as diffuse interstitial lung disease with bilateral hilar lymphadenopathy. Furthermore, histologic examination of lesions demonstrates classic noncaseating granulomas containing epithelioid cells, multinucleated giant cells with inclusion bodies, and lymphocytes. ${ }^{11}$

A skin biopsy is required to establish the diagnosis of MRH. In general, patients with MRH and no underlying malignancy have a good prognosis and respond to anti-inflammatory therapies such as nonsteroidal antiinflammatory drugs and corticosteroids. Other agents including methotrexate, cyclophosphamide, and tumor necrosis factor $\alpha$ inhibitors also have been effective in more severe cases. ${ }^{1,3,12}$ Finally, in addition to treating the cutaneous manifestations of MRH, it is important to screen patients for underlying malignancies and other autoimmune conditions.

\section{REFERENCES}

1. Tajirian AL, Malik MK, Robinson-Bostom L, et al. Multicentric reticulohistiocytosis. Clin Dermatol. 2006;24:486-492.

2. Gold RH, Metzger AL, Mirra JM, et al. Multicentric reticulohistiocytosis (lipoid dermato-arthritis). an erosive polyarthritis with distinctive clinical, roentgenographic and pathologic features. Am J Roentgenol Radium Ther Nucl Med. 1975;124:610-624.

3. Luz FB, Gaspar TAP, Kalil-Gaspar N, et al. Multicentric reticulohistiocytosis. J Eur Acad Dermatol Venereol. 2001;15:524-531.

4. Barrow MV. The nails in multicentric reticulohistiocytosis. (lipoid dermato-arthritis). Arch Dermatol. 1967;95:200-201.

5. Barrow MV, Holubar K. Multicentric reticulohistiocytosis. a review of 33 patients. Medicine (Baltimore). 1969;48:287-305.

6. Snow JL, Muller SA. Malignancy-associated multicentric reticulohistiocytosis: a clinical, histological and immunophenotypic study. Br J Dermatol. 1995;133:71-76.

7. Yiannias JA, el-Azhary RA, Gibson LE. Erythema elevatum diutinum: a clinical and histopathologic study of 13 patients. J Am Acad Dermatol. 1992;26:38-44.

8. Smith ES, Hallman JR, DeLuca AM, et al. Dermatomyositis: a clinicopathological study of 40 patients. Am J Dermatopathol. 2009; 31:61-67.

9. Athanasou NA, Quinn J, Woods CG, et al. Immunohistology of rheumatoid nodules and rheumatoid synovium. Ann Rheum Dis. 1988;47:398-403.

10. Yanardag H, Pamuk ON, Karayel T. Cutaneous involvement in sarcoidosis: analysis of the features in 170 patients. Respir Med. 2003;97:978-982.

11. MaY, Gal A, Koss MN. The pathology of pulmonary sarcoidosis: update. Semin Diagn Pathol. 2007;24:150-161.

12. Kovach BT, Calamia KT, Walsh JS, et al. Treatment of multicentric reticulohistiocytosis with etanercept. Arch Dermatol. 2004;140:919-921. 